\title{
Pretargeted Immuno-PET: Overcoming Limitations of Space and Time
}

\author{
Bernadette V. Marquez and Suzanne E. Lapi \\ Department of Radiology, University of Alabama at Birmingham, Birmingham, Alabama
}

\begin{abstract}
A
lthough the development of radiolabeled antibodies for preclinical and clinical use in PET imaging continues to mature into a widespread technique (1-4), limitations still remain: namely space, in which the antigens to which our antibody is targeting are located (intracellularly or cell surface, bound or circulating), and time, the extended period after which the imaging must take place, to allow for blood clearance of the long-circulating antibody. Typically, circulating antigens can be overcome by a predose of cold antibody to saturate these off-target sites, whereas limitations in timing can be overcome by simply imaging at longer time points. The widespread availability of long-lived radionuclides in the field of nuclear medicine such as ${ }^{89} \mathrm{Zr}$ and ${ }^{124} \mathrm{I}$ have enabled the latter. However, the main drawback in direct imaging of radiolabeled antibodies with such radionuclides is that the patient is exposed to radiation longer than traditional radionuclides, resulting in greater amounts of accumulated absorbed dose to normal tissues.
\end{abstract}

\section{See page 453}

Pretargeted PET circumvents prolonged radiation exposure by physically separating the targeting antibody from the radiotracer, until most of the antibody accumulates in the tumor and the unbound antibody clears from the bloodstream. In this approach, the antibody is modified as a bispecific targeting agent, which binds a marker on the surface of cancer cells and its radioligand. Pretargeting is a 2- or 3-step process. The bispecific antibody 1) is first administered in vivo to accumulate in the tumor and is allowed to clear from the bloodstream naturally, or 2) is sequestered by injection of a clearing agent to increase the rate of clearance. This first step can take several days, and the assumption is that the antibody persists on the cancer cell surface for 3 ) the radioligand to bind or a minimal amount of the antibody is internalized. The unbound radioligand will clear rapidly from the bloodstream because of its small size. In this case, relatively short-lived radionuclides may be used such as ${ }^{64} \mathrm{Cu}$ (half-life, $12.7 \mathrm{~h}$ ) or ${ }^{18} \mathrm{~F}$ (half-life, $1.8 \mathrm{~h}$ ).

Received Nov. 16, 2015; revision accepted Nov. 17, 2015

For correspondence or reprints contact: Suzanne Lapi, University of Alabama at Birmingham, Wallace Tumor Institute 310F, 1720 2nd Ave., South Birmingham, AL 35294.

E-mail: lapi@uab.edu

Published online Dec. 10, 2015.

COPYRIGHT (C) 2016 by the Society of Nuclear Medicine and Molecular Imaging, Inc.

DOI: $10.2967 /$ jnumed.115.168096
PET imaging can then be performed as early as the same day, reducing radiation exposure to the patient.

The concept of pretargeting has previously been introduced as a technique to improve targeted radiotherapy using radiolabeled antibodies by reducing exposure to normal organs while retaining tumor accumulation. The first was an anti-DOTA-metal complex antibody with the rationale that antibodies can be humanized to prevent immunogenicity and the DOTA-radiometal complex will bind to its antibody fusion protein whereas the unbound portion is small enough to clear rapidly from the bloodstream (5). Building on the potential of the anti-DOTA-metal chelate strategy, several pretargeted therapeutic and imaging agents have been developed, each with different chemistries to optimize the binding affinity and clearance of both radioligand and tumor-targeting antibody partner (6). However, important disadvantages to these systems are that they are not widely available to the field of nuclear medicine as a whole. In some cases, they are also difficult and expensive to produce, limiting the widespread application of these technologies. In recent years, much smaller pretargeting agents using bioorthogonal click chemistry has gained significant attention because of the commercial availability of these agents, the high rate of reaction, and the low probability of eliciting an immunogenic response, which led to much simpler production and overall effectiveness of pretargeting in vivo ( 6 ).

The study in this issue of The Journal of Nuclear Medicine by Houghton et al. (7) capitalizes on these advantages of bioorthogonal click chemistry between an antibody and its radiotracer to challenge issues of antibody internalization and circulating antigens. Work in this area has previously investigated the use of transcyclooctene (TCO) and tetrazine (Tz) bioorthogonal click technique (8), previously showing the utility of this strategy in vivo. This new work expands on the previous groundwork by applying this pretargeting approach to imaging of carbohydrate antigen 19.9 (CA19.9), a known marker of metastasis in pancreatic ductal adenocarcinoma and previously imaged using radiolabeled intact antibodies and engineered antibody fragments (9-11). This antigen is overexpressed in $90 \%$ of pancreatic cancer cases, whereas it is minimally expressed in normal tissues. CA19.9 can shed from the surface of cancer cells and also quickly internalizes the antibody to which it binds. Although the shed antigen can be quantified from blood and is currently being used in the clinic as a prognostic biomarker, these levels may not be representative of its expression levels in the tumor. Thus, pretargeted PET imaging of this important biomarker may help with treatment decisions that involve targeting of this antigen or its pathway, while reducing radiation exposure to the patient.

In the study by Houghton et al., the authors used a previously studied antibody, 5B1, modified with the TCO moiety, to undergo 
a click reaction with a radiolabeled, PEGylated $\mathrm{Tz},{ }^{64} \mathrm{Cu}-\mathrm{NOTA}-$ $\mathrm{PEG}_{7}-\mathrm{Tz}$, in vivo. The previously investigated ${ }^{64} \mathrm{Cu}-\mathrm{NOTA}-\mathrm{Tz}$ and its properties were compared with the pharmacokinetic properties of the PEGylated construct. The authors used xenograft models with orthotopic and subcutaneous tumors generated from pancreatic cancer cell lines known to shed CA19.9 and internalize 5B1 rapidly, which they confirmed by conducting cellular internalization assays in vitro. PET imaging studies were conducted with varying intervals between the injections of the 5B1-TCO conjugate and $\mathrm{Tz}$ radiotracer pair. A lag time of $72 \mathrm{~h}$ was found to lead to better images than a 48-h interval, likely due to the lengthy blood circulation time of the intact antibody. At this time interval, tumors were visualized as early as $6 \mathrm{~h}$ after injection of the ${ }^{64} \mathrm{Cu}-\mathrm{NOTA}-\mathrm{PEG}_{7}-\mathrm{Tz}$, with greater tumor-to-digestive tract uptake ratios than with the ${ }^{64} \mathrm{Cu}-\mathrm{NOTA}-\mathrm{Tz}$ radiotracer. In contrast, a 120-h time interval led to decreased tumor accumulation, likely due to internalization of the antibody at this extended time point.

The authors optimized the timing of pretargeting strategically to detect CA19.9 in the tumor. As the authors noted, more investigations are needed to determine the rate of antigen recycling and extent of antibody internalization, as well as the degree of antigen shedding as a function of antibody binding in vivo. This information will be relevant during serial pretargeted PET imaging over the course of a potential anti-CA19.9 antibody therapy. It will be important to determine whether the optimized lag and imaging times will still apply over the course of therapy. Nevertheless, the simple pegylation of the radiotracer significantly improved its biodistribution properties to enhance the image quality within a relatively short time frame.

Additionally, dosimetry studies showed a significant reduction ( $>25$ fold) in the total-body dose, compared with studies conducted with ${ }^{89} \mathrm{Zr}$-labeled DFO-5B1, which the authors noted is currently in the process of clinical translation. One might think that comparing dosimetry between these 2 different radioisotopes with obvious differences in decay properties might be similar to comparing apples and oranges. However, one should consider the big picturethat the dosimetry comparison is between 2 imaging agents that directly $\left({ }^{89} \mathrm{Zr}-5 \mathrm{~B} 1\right)$ or indirectly $\left(5 \mathrm{~B} 1-\mathrm{TCO}+{ }^{64} \mathrm{Cu}-\mathrm{Tz}\right)$ target CA19.9. An example of this significant difference is the dramatic reduction of absorbed radiation dose to the liver (54-fold) using the pretargeting approach. The liver is the clearance organ for monoclonal antibodies and is often the dose-limiting organ. The pretargeting agents in this study showed their advantage over direct imaging of radiolabeled antibodies in the reduction of the total radiation exposure while providing high-quality images of the target.

Although this strategy ultimately leads to specific imaging of CA19.9, this should be considered a complementary approach to imaging with intact antibodies, if the treatment plan involves targeting CA19.9 with monoclonal antibodies. When designing a molecular imaging study, one needs to think carefully about the biologic question being examined: detection or quantification of the imaging target/receptor in the region of interest, or tracking or quantification of the drug or imaging agent in the region of interest. Pretargeted PET imaging strategies may be an excellent technique for answering the first, but direct PET imaging of radiolabeled antibodies or antibody drug conjugates is still an essential tool for answering the latter.

Overall, the well-designed work by Houghton et al. (7) adds a new perspective to the field of pretargeting and illustrates that internalizing antibodies and circulating antigens are not barriers to this approach. This strategy paves the way toward other antibody-target combinations, targeted radiotherapy to give a pretargeted theranostic agents, and its application in other CA19.9overexpressing diseases.

\section{DISCLOSURE}

No potential conflict of interest relevant to this article was reported.

\section{REFERENCES}

1. Oosting SF, Brouwers AH, van Es SC, et al. ${ }^{89} \mathrm{Zr}$-bevacizumab PET visualizes heterogeneous tracer accumulation in tumor lesions of renal cell carcinoma patients and differential effects of antiangiogenic treatment. J Nucl Med. 2015; $56: 63-69$.

2. Sham JG, Kievit FM, Grierson JR, et al. Glypican-3-targeted ${ }^{89} \mathrm{Zr}$ PET imaging of hepatocellular carcinoma. J Nucl Med. 2014;55:799-804.

3. Pandit-Taskar N, O'Donoghue JA, Durack JC, et al. A phase I/II study for analytic validation of ${ }^{89} \mathrm{Zr}$-J591 immunoPET as a molecular imaging agent for metastatic prostate cancer. Clin Cancer Res. 2015;21:5277-5285.

4. Marquez BV, Ikotun OF, Zheleznyak A, et al. Evaluation of ${ }^{89} \mathrm{Zr}$-pertuzumab in breast cancer xenografts. Mol Pharm. 2014;11:3988-3995.

5. Reardan DT, Meares CF, Goodwin DA, et al. Antibodies against metal chelates. Nature. 1985;316:265-268.

6. Knight JC, Cornelissen B. Bioorthogonal chemistry: implications for pretargeted nuclear (PET/SPECT) imaging and therapy. Am J Nucl Med Mol Imaging. 2014;4: 96-113.

7. Houghton JL, Zeglis BM, Abdel-Atti D, Sawada R, Scholz WW, and Lewis JS. Pretargeted immuno-PET of pancreatic cancer: overcoming circulating antigen and internalized antibody to reduce radiation doses. J Nucl Med. 2016;57:453-459.

8. Zeglis BM, Mohindra P, Weissmann GI, et al. Modular strategy for the construction of radiometalated antibodies for positron emission tomography based on inverse electron demand Diels-Alder click chemistry. Bioconjug Chem. 2011;22:2048-2059.

9. Girgis MD, Olafsen T, Kenanova V, McCabe KE, Wu AM, Tomlinson JS. CA19-9 as a potential target for radiolabeled antibody-based positron emission tomography of pancreas cancer. Int J Mol Imaging. 2011;2011:834515.

10. Girgis MD, Kenanova V, Olafsen T, McCabe KE, Wu AM, Tomlinson JS. AntiCA19-9 diabody as a PET imaging probe for pancreas cancer. J Surg Res. 2011;170: $169-178$.

11. Viola-Villegas NT, Rice SL, Carlin S, et al. Applying PET to broaden the diagnostic utility of the clinically validated CA19.9 serum biomarker for oncology. J Nucl Med. 2013;54:1876-1882. 